\title{
BORDISM OF SEMIFREE CIRCLE ACTIONS ON SPIN MANIFOLDS
}

\author{
LUCILIA DARUIZ BORSARI
}

\begin{abstract}
Using traditional methods in bordism theory, an almost complete description of the rational bordism groups of semifree circle actions on Spin manifolds is given. The single remaining problem, to describe the ideal of $\Omega_{*}^{\text {Spin }} \otimes \mathbf{Q}$ generated by bordism classes of Spin manifolds admitting a semifree action of odd type, has been recently solved by $\mathrm{S}$. Ochanine [O].
\end{abstract}

1. Introduction. In this work we study bordism groups of semifree circle actions on Spin manifolds.

The motivation for studying these groups comes from a question of E. Witten (see [W]) who is interested in the Dirac operator with coefficients in the tangent bundle of a Spin manifold $M^{2 n}$. Its ordinary index is given by $\left\langle\hat{\mathscr{A}}(M) \operatorname{ch}(T), \sigma_{M}\right\rangle$, where $\hat{\mathscr{A}}(M)$ is the total $\hat{A}$-class of the tangent bundle of $M$, and $\operatorname{ch}(T)$ denotes the Chern character of the complexification of the tangent bundle of $M$. Witten asks if the character valued index, for an $S^{1}$-action on a Spin manifold, of this operator is a constant. He suggested that the problem could be explored via the bordism groups of circle actions on Spin manifolds.

In order to simplify the problem, we restrict our attention to semifree circle actions.

Our main result, Theorem 2.4, concerns the comparison of the Spin bordism groups of semifree circle actions with their oriented analogues, after tensoring with the rationals. We complete the bordism analysis except for the determination of the ideal $I_{*}$ in $\Omega_{*}^{\text {Spin }} \otimes \mathbf{Q}$ consisting of multiples of bordism classes of complex projective space bundles of even dimensional complex vector bundles. This ideal is examined in $\S 3$, and has recently been determined by $\mathrm{S}$. Ochanine (see [O]).

I wish to thank my thesis advisor, Professor Peter Landweber, for his ideas, concern and support. I am also indebted to Professor R. E. Stong whose writings have given me many elucidative ideas and much encouragement. I also thank Professor V. Young for very helpful conversations.

These results form part of the author's 1985 Ph.D. thesis [B], written at Rutgers University. The author is grateful to the National Science Foundation for summer support.

Received by the editors April 30, 1986.

1980 Mathematics Subject Classification (1985 Revision). Primary 57R85; 57R20.

(C)1987 American Mathematical Society $0002-9947 / 87 \$ 1.00+\$ .25$ per page 
2. Bordism analysis. We start with some definitions and notations.

Let $B$ be a $C W$-complex and $\xi:(E(\xi), p, B)$ a principal $S O(n)$-bundle. A Spin structure on $\xi$ is a pair $(\eta, f)$ consisting of

(1) a principal $\operatorname{Spin}(n)$-bundle $\eta$ over $B$; and

(2) a map $f: E(\eta) \rightarrow E(\xi)$ such that the following diagram commutes:

$$
\begin{array}{cccc}
E(\eta) \times \operatorname{Spin}(n) & \rightarrow & E(\eta) & \\
\downarrow f \times \phi_{n} & & \downarrow f & { }^{B} \\
E(\xi) \times S O(n) & \rightarrow & E(\xi)
\end{array}
$$

Here $\phi_{n}$ denotes the standard homomorphism from $\operatorname{Spin}(n)$ to $S O(n)$.

DEFINITION. A Spin manifold is an oriented Riemannian manifold together with a Spin structure on its bundle of oriented frames.

A smooth circle action on an oriented smooth manifold is said to be semifree if all isotropy groups are either $S^{1}$ or the unit subgroup of $S^{1}$.

We have, as usual, a long exact sequence

$$
\cdots \rightarrow S F_{n}^{\text {Spin }} \stackrel{j}{\rightarrow} M_{n}^{\text {Spin }} \stackrel{\partial}{\rightarrow} F_{n-1}^{\text {Spin }} \stackrel{i}{\rightarrow} S F_{n-1}^{\text {Spin }} \rightarrow \cdots,
$$

where

(a) $F_{n}^{\text {Spin }}$ denotes the bordism group of free circle actions on closed $n$-dimensional Spin manifolds,

(b) $S F_{n}^{\text {Spin }}$ denotes the bordism group of semifree circle actions on closed $n$-dimensional Spin manifolds, and

(c) $M_{n}^{\text {Spin }}$ denotes the bordism group of semifree circle actions on compact $n$-dimensional Spin manifolds which are free on the boundary.

The maps $i, j$ and $\partial$ are the usual ones (see [C]).

The relative group $M_{n}^{\text {Spin }}$. Let $\gamma_{k}$ denote the universal complex vector bundle over $B U_{k}$. Denote by $\Omega_{n}^{\text {Spin }}\left(B U_{k}, \gamma_{k}\right)$ the bordism group of triples $(M, f, s)$, where $M$ is an oriented ( $n-2 k$ )-dimensional manifold, $f: M \rightarrow B U_{k}$ is continuous, and $s$ is a Spin structure on $\tau M \oplus f^{*}\left(\gamma_{k}\right)$.

Given $[W, \alpha] \in M_{n}^{\text {Spin }}$, let $X^{n-2 k}$ denote the union of the $(n-2 k)$-dimensional components of the fixed point set of $\alpha$. Consider $f_{k}: X^{n-2 k} \rightarrow B U_{k}$, the classifying map for the normal bundle $\nu_{k}$ to $X^{n-2 k}$. If $s$ denotes the Spin structure on $W$, then, since $(\tau W) \mid X^{n-2 k}=\tau X^{n-2 k} \oplus f_{k}^{*}\left(\gamma_{k}\right)=\tau X^{n-2 k} \oplus \nu_{k}, s$ induces a Spin structure $s_{k}$ on $\tau X^{n-2 k} \oplus f_{k}^{*}\left(\gamma_{k}\right)$.

Define

$$
\rho_{n}: M_{n}^{\text {Spin }} \rightarrow \bigoplus_{k} \Omega_{n}^{\text {Spin }}\left(B U_{k}, \gamma_{k}\right)
$$

by

$$
\rho_{n}([W, \alpha])=\bigoplus_{k}\left[X^{n-2 k}, f_{k}, s_{k}\right] .
$$


Proposition 2.1. The homomorphism $\rho_{n}$ defined above is an isomorphism.

Proof. We exhibit an inverse for $\rho_{n}$. Let $(M, f, s)$ represent an element in $\Omega_{n}^{\text {Spin }}\left(B U_{k}, \gamma_{k}\right)$ and put $E=f^{*}\left(\gamma_{k}\right)$; then $f$ induces a map on disk bundles $\tilde{f}$ : $D(E) \rightarrow D\left(\gamma_{k}\right)$, which we may assume transverse regular to $B U_{k}$. Then $\nu(M, E)=$ $f^{*}\left(\nu\left(B U_{k}, \gamma_{k}\right)\right)=f^{*}\left(\gamma_{k}\right)=E$, where $\nu$ denotes normal bundle. Since $\tau E=$ $p^{*}(\tau M \oplus E)$, where $p: E \rightarrow M$ is the projection, $s$ induces a Spin structure on $\tau E$ and so on $D(E)$. Let $\beta$ denote complex multiplication on $D(E)$.

The correspondence $(M, f, s) \rightarrow(D(E), \beta)$ gives an inverse for $\rho_{n}$.

REMARK. Using the same techniques as in $[\mathbf{L}-\mathbf{W}]$ one can show that

$$
\Omega_{*}^{\text {Spin }}\left(B U_{k}, \gamma_{k}\right) \cong \Omega_{*}^{\text {Spin }}\left(D\left(\gamma_{k}\right), S\left(\gamma_{k}\right)\right)
$$

and therefore

$$
M_{n}^{\text {Spin }} \otimes \mathbf{Q} \cong \bigoplus_{k} \Omega_{n-2 k}\left(B U_{k}\right) \otimes \mathbf{Q}
$$

In particular, $M_{n}^{\text {Spin }} \otimes \mathbf{Q}=0$ for odd $n$.

The group $F_{n}^{\text {Spin }}$. In order to describe these groups we need to make the distinction between actions of even and odd types. Let $M^{n}$ be a connected Spin manifold. Denote by $Q$ the bundle of oriented frames and by $P$ the principal $\operatorname{Spin}(n)$-bundle over $M$. Let $\alpha: S^{1} \times M \rightarrow M$ be a smooth circle action. Then $\alpha$ induces a circle action on $Q$ that can be viewed as a one parameter family of maps $\alpha_{t}: Q \rightarrow Q$, $t \in \mathbf{R}$. Also, $\alpha_{0}=\alpha_{1}=$ identity on $Q$. Since $P$ is a double covering of $Q, \alpha_{t}$ lifts to $\tilde{\alpha}_{t}: P \rightarrow P$ with $\tilde{\alpha}_{0}=$ identity on $P$ and $\tilde{\alpha}_{1}$ either the identity on $P$ or multiplication by $-1 \in \mathbf{Z}_{2}=\operatorname{Ker}\{\operatorname{Spin}(n) \rightarrow S O(n)\}$.

If $\tilde{\alpha}_{1}=$ identity on $P$, then $\tilde{\alpha}_{t}$ induces a circle action on $P$ which commutes with the right action of $\operatorname{Spin}(n)$ on $P$ and is compatible with $u: P \rightarrow Q$. In this case we say that $\alpha$ is of even type.

If $\tilde{\alpha}_{1}=$ multiplication by $-1 \in \mathbf{Z}_{2} \subset \operatorname{Spin}(n)$, then $\tilde{\alpha}_{t}$ induces an action of $\hat{S}^{1}$, the connected double covering of $S^{1}$, on $P$ with the same properties as above. We then call $\alpha$ of odd type (see [A-H]).

The next two propositions characterize free circle actions of even and odd types. Their proofs will be presented in $\$ 4$.

Proposition 2.2. Let $M^{n}$ be a connected Spin manifold and let $\alpha: S^{1} \times M \rightarrow M$ be a smooth free circle action on $M$. Then $\alpha$ is of even type if and only if the orbit space $M / S^{1}$ admits a Spin structure which induces via $\pi: M \rightarrow M / S^{1}$ the given Spin structure on $M$.

Proposition 2.3. Let $M^{n}$ be a connected Spin manifold and $\alpha: S^{1} \times M \rightarrow M$ be a smooth free circle action on $M$. Then $\alpha$ is of odd type if and only if the orbit space $M / S^{1}$ can be given a Spinc structure, with principal $U(1)$-bundle $M \stackrel{\pi}{\rightarrow} M / S^{1}$ and principal $\operatorname{Spin}^{c}(n)$-bundle given by the composition of the principal $\operatorname{Spin}(n)$-bundle over $M$ and $\pi$.

Notice that we may write $F_{n}^{\text {Spin }}$ as a direct sum

$$
F_{n}^{\text {Spin }}=F_{n}^{\text {Spin,ev }} \oplus F_{n}^{\text {Spin,odd }}
$$


where the direct summands are bordism groups of free circle actions of even or odd types, respectively.

Translating the last two propositions to bordism language, we obtain isomorphisms

$$
\begin{aligned}
\mu_{n}: \quad & F_{n}^{\text {Spin }, \text { odd }} \rightarrow \Omega_{n-1}^{\operatorname{Spin}^{c}} \\
& {[M, \alpha] \rightarrow\left[M / S^{1}\right] }
\end{aligned}
$$

and

$$
\begin{aligned}
\sigma_{n}: & F_{n}^{\text {Spin }, \mathrm{ev}} \rightarrow \Omega_{n-1}^{\text {Spin }}\left(\mathbf{C} P^{\infty}\right) \\
{[M, \alpha] } & \rightarrow\left[M / S^{1}, f\right]
\end{aligned}
$$

where $f: M / S^{1} \rightarrow \mathrm{C} P^{\infty}$ classifies the complex line bundle associated with $M \rightarrow$ $M / S^{1}$.

REMARK. Since $\Omega_{n}^{\mathrm{Spin}^{c}} \otimes \mathbf{Q} \cong \Omega_{n}\left(\mathbf{C} P^{\infty}\right) \otimes \mathbf{Q} \cong \Omega_{n}^{\mathrm{Spin}}\left(\mathbf{C} P^{\infty}\right) \otimes \mathbf{Q}$ (see [St]), we conclude that $F_{n}^{\text {Spin }} \otimes \mathbf{Q}=0$ for even $n$.

We also have the following:

A semifree circle action on a Spin manifold with nonempty fixed point set is of even (resp. odd) type if and only if the codimension of all components of the fixed point set are congruent to zero (resp. 2) mod 4. Indeed, Atiyah and Bott (see [A-B]) prove the same result for involutions preserving a Spin structure. Since $M^{\mathbf{Z}_{2}}=M^{S^{1}}$ for a semifree circle action, our assertion follows at once.

We now state and prove our main result about the groups $S F_{n}^{\text {Spin }}$.

THEOREM 2.4. Let $S F_{n}$ denote the bordism group of semifree circle actions on closed oriented manifolds. Let $\phi_{S F}: S F_{n}^{\text {Spin }} \rightarrow S F_{n}$ be the forgetful homomorphism. Then

(a) the map $\phi_{S F}$ is injective in even dimensions after tensoring with the rationals.

(b) a multiple of $[M, \alpha] \in S F_{2 n}$ is in the image of $\phi_{S F}$ if and only if

$$
\bigoplus_{k \text { even }}\left[\mathbf{C P}\left(\nu_{k}\right), \lambda_{k}\right]=\bigoplus_{k \text { odd }}\left[\mathbf{C P}\left(\nu_{k}\right), \lambda_{k}\right]=0
$$

in $\Omega_{2 n-2}\left(\mathbf{C P} P^{\infty}\right) \otimes \mathbf{Q}$.

Here $\nu_{k}$ denotes the normal bundle to the union of the $(n-2 k)$-dimensional components of the fixed point set of $\alpha$, and $\lambda_{k} \rightarrow \mathbf{C P}\left(\nu_{k}\right)$ is the canonical line bundle over the complex projective space bundle, $\mathrm{CP}\left(\nu_{k}\right)$, of $\nu_{k}$.

We need the following lemma.

LEMMA 2.5. The map $F_{2 n-1}^{\text {Spin,odd }} \stackrel{i}{\rightarrow} S F_{2 n-1}^{\text {Spin,odd }}$ is trivial.

Proof. Let $(M, \alpha)$ represent an element in $F_{2 n-1}^{\text {Spinodd }}$ and let $D^{2}$ be the 2-dimensional disk. Then $\alpha \times \beta$ is a free $S^{1}$-action on $M \times D^{2}$, where $\beta$ is complex multiplication on $D^{2}$. The Spin structures on $M$ and $D^{2}$ induce a Spin structure on $M \times D^{2}$ for which $\alpha \times \beta$ has even type. Therefore $W=\left(M \times D^{2}\right) / S^{1}$ is a Spin manifold whose boundary can be identified with $M$ as Spin manifolds. If we let the circle act on $W$, by acting on the first factor via $\alpha$, we obtain a semifree circle action on $W$ having odd type. 
Proof of Theorem 2.4. We denote by $\mathbf{Q} S F_{n}^{\text {Spin }}, \mathbf{Q} F_{n}^{\text {Spin }}$ and $\mathbf{Q} M_{n}^{\text {Spin }}$ the groups $S F_{n}^{\text {Spin }}, F_{n}^{\text {Spin }}$ and $M_{n}^{\text {Spin }}$ tensored with the rationals.

From the results about these groups, we have the following exact sequence

$$
0 \rightarrow \mathbf{Q S} F_{2 n}^{\text {Spin }} \rightarrow \mathbf{Q} M_{2 n}^{\text {Spin }} \rightarrow \mathbf{Q} F_{2 n-1}^{\text {Spin }} \stackrel{i}{\rightarrow} \mathbf{Q} S F_{2 n-1}^{\text {Spin,ev }} \rightarrow 0 .
$$

Also, for oriented semifree bordism, there is a short exact sequence (see [U])

$$
0 \rightarrow S F_{2 n} \stackrel{j_{*}}{\rightarrow} \bigoplus_{k} \Omega_{2 n-2 k}\left(B U_{k}\right) \stackrel{\partial_{*}}{\rightarrow} \Omega_{2 n-2}\left(\mathbf{C} P^{\infty}\right) \rightarrow 0
$$

where $j_{*}([M, \alpha])=\oplus_{k}\left[\nu_{k}\right]$, for $\nu_{k}$ the normal bundle to the union of the $(2 n-2 k)$ dimensional components of the fixed point set of $\alpha$. The map $\partial_{*}$ associates to each bordism class of a complex vector bundle $\xi$ the element $[\mathbf{C P}(\xi), \lambda]$, where $\lambda \rightarrow \mathbf{C P}(\xi)$ is the complex line bundle.

Using the descriptions of the groups $M_{n}^{\text {Spin }}$ and $F_{n}^{\text {Spin }}$ we have the following exact sequence:

$$
0 \rightarrow \mathbf{Q} S F_{2 n}^{\mathrm{Spin}} \stackrel{j^{*}}{\rightarrow} \bigoplus_{k} \Omega_{2 n}^{\mathrm{Spin}}\left(B U_{k}, \gamma_{k}\right) \otimes \mathbf{Q} \stackrel{\partial}{\rightarrow} \Omega_{2 n-2}^{\mathrm{Spin}^{c}} \otimes \mathbf{Q} \oplus \operatorname{Ker} i_{\mathrm{ev}}^{*} \rightarrow 0,
$$

where $i_{\mathrm{ev}}^{*}$ is the composition

$$
\Omega_{2 n-2}^{\text {Spin }}\left(\mathbf{C} P^{\infty}\right) \otimes \mathbf{Q} \cong \mathbf{Q} F_{2 n-1}^{\text {Spin,ev }} \rightarrow \mathbf{Q S} F_{2 n-1}^{\text {Spin,ev }} .
$$

Since elements of $\Omega_{2 n}^{\mathrm{Spin}}\left(B U_{k}, \lambda_{k}\right)$ can be viewed as bordism classes of $k$-plane bundles with a Spin structure on their total spaces, the maps $j^{*}$ and $\partial^{*}$ are defined as in the oriented case. Also, $\partial^{*}$ maps $\oplus_{k}$ even $\Omega_{2 n}^{\operatorname{Spin}}\left(B U_{k}, \gamma_{k}\right)$ to $\operatorname{ker} i_{\mathrm{ev}}^{*}$ and, $\oplus_{k \text { odd }} \Omega_{2 n}^{\text {Spin }}\left(B U_{k}, \gamma_{k}\right)$ to $\Omega_{2 n-2}^{\operatorname{Spin}^{c}}$.

Consider the following commutative diagram:

$$
\begin{aligned}
& 0 \rightarrow \mathbf{Q S F} F_{2 n}^{\mathrm{Spin}} \rightarrow \bigoplus_{k} \Omega_{2 n}^{\mathrm{Spin}}\left(B U_{k}, \gamma_{k}\right) \otimes \mathbf{Q} \quad \rightarrow \quad\left(\Omega_{2 n-2}^{\mathrm{Spin}^{c}} \oplus \operatorname{ker} i_{\mathrm{ev}}^{*}\right) \otimes \mathbf{Q} \rightarrow 0 \\
& \downarrow \phi_{S F} \quad \downarrow \phi_{\text {rel }} \quad \downarrow \phi_{F} \\
& 0 \rightarrow \mathbf{Q S F} F_{2 n} \rightarrow \bigoplus_{k} \Omega_{2 n-2 k}\left(B U_{k}\right) \otimes \mathbf{Q} \quad \rightarrow \quad \Omega_{2 n-2}\left(\mathbf{C} P^{\infty}\right) \otimes \mathbf{Q} \quad \rightarrow 0 \text {, }
\end{aligned}
$$

where the vertical arrows are forgetful homomorphisms.

Since $\phi_{\text {rel }}$ is an isomorphism, the map $\phi_{S F}$ is injective.

Next, let $[M, \alpha] \in \mathbf{Q} S F_{2 n}$. Write $j^{*}([M, \alpha])=\oplus_{k}\left[\nu_{k}\right]$ and put $\left[\xi_{k}\right]=\phi_{\text {rel }}^{-1}\left(\left[\nu_{k}\right]\right)$. Then $[M, \alpha]$ lies in the image of $\phi_{S F}$ if and only if $\oplus_{k \text { even }} \partial *\left[\xi_{k}\right]=0$ in $\operatorname{ker} i_{\text {ev }}^{*}$, and $\oplus_{k \text { odd }} \partial^{*}\left[\xi_{k}\right]=0$ in $\Omega_{2 n-2}^{\mathrm{Spin}^{c}} \otimes \mathbf{Q}$. But this condition is equivalent to having

$$
\bigoplus_{k \text { even }}\left[\mathbf{C P}\left(\nu_{k}\right), \lambda_{k}\right]=\bigoplus_{k \text { odd }}\left[\mathbf{C P}\left(\nu_{k}\right), \lambda_{k}\right]=0
$$

in $\Omega_{2 n-2}\left(\mathbf{C} P^{\infty}\right) \otimes \mathbf{Q}$.

3. The ideal $I_{*}$. The distinction between the semifree Spin bordism groups and semifree oriented groups lies in the fact that the map $i_{\mathrm{ev}}: F_{2 n-1}^{\text {Spin,ev }} \rightarrow S F_{2 n-1}^{\text {Spin,ev }}$ is not trivial.

From the exactness of sequence (1), we see that ker $i_{\mathrm{ev}}$ coincides with the image of the map

$$
\partial^{*}: \bigoplus_{k} \Omega_{2 n}^{\mathrm{Spin}}\left(B U_{2 k}, \gamma_{2 k}\right) \rightarrow \Omega_{2 n-2}^{\mathrm{Spin}}\left(\mathbf{C} P^{\infty}\right)
$$


Rationally, this map can be viewed as a map from $\oplus_{k} \Omega_{2 n-4 k}\left(B U_{2 k}\right) \otimes \mathbf{Q}$ to $\Omega_{2 n-2}\left(\mathbf{C} P^{\infty}\right) \otimes \mathbf{Q}$. If we let $x_{k}=\left[\eta_{k} \oplus \varepsilon^{2 k-1} \rightarrow \mathbf{C} P^{n-2 k}\right]$, where $\eta_{k}$ is the canonical line bundle and $\varepsilon^{2 k-1}$ is the $(2 k-1)$-dimensional trivial bundle, it can be seen that $\left\{\partial^{*}\left(x_{k}\right)\right\}$ forms a basis of $\tilde{\Omega}_{2 n-2}\left(\mathbf{C} P^{\infty}\right) \otimes \mathbf{Q}$ (see [C-F]).

We then proceed to analyze the image of the composition $\oplus_{k} \Omega_{4 *+2}^{\text {Spin }}\left(B U_{2 k}, \gamma_{2 k}\right) \otimes$ $\mathbf{Q} \stackrel{\partial^{*}}{\rightarrow} \Omega_{4 *}^{\text {Spin }}\left(\mathbf{C} P^{\infty}\right) \otimes \mathbf{Q} \stackrel{\varepsilon}{\rightarrow} \Omega_{4 *}^{\text {Spin }} \otimes \mathbf{Q}$. Denote by $I_{*}$ the image of $\varepsilon^{\circ} \partial^{*} ;$ then $I_{*}$ is an ideal of $\Omega_{*}^{\text {Spin }} \otimes \mathbf{Q}$.

Let $J_{*}$ be the ideal of $\Omega_{*}^{\text {Spin }} \otimes \mathbf{Q}$ consisting of multiples of bordism classes of Spin manifolds which admit a semifree circle action of odd type.

Proposition 3.1. As ideals of $\Omega_{*}^{\text {Spin }} \otimes \mathbf{Q}, I_{*}$ and $J_{*}$ agree.

Proof. Let us show first that $J_{*} \subset I_{*}$. Start with a Spin manifold $M^{n}$ with a semifree $S^{1}$ action of odd type $\alpha$. Let $\nu_{k}$ denote the normal bundle to the union of the $(n-2 k)$-dimensional components of the fixed point set of $\alpha$. By [U], we have $\left[M^{n}\right]=\oplus_{k}\left[\mathbf{C P}\left(\nu_{k} \oplus \varepsilon\right)\right]$ in $\Omega_{n}$. Since $\alpha$ has odd type, $\nu_{k} \oplus \varepsilon$ is even dimensional and its total space admits a Spin structure. Hence, the sphere bundle of $\nu_{k} \oplus \varepsilon$ is a Spin manifold which bounds the disk bundle of $\nu_{k} \oplus \varepsilon$ as Spin manifolds. Therefore, complex multiplication on the sphere bundle of $\nu_{k} \oplus \varepsilon$ is of even type. So, $\left[M^{n}\right]=$ $\oplus_{k}\left[\mathbf{C P}\left(\nu_{k} \oplus \varepsilon\right)\right]$ in $\Omega_{*}^{\text {Spin }} \cap \mathbf{Q}$, which proves that $J_{*} \subset I_{*}$.

To obtain the other inclusion, we notice that given $\xi$ an even dimensional complex vector bundle representing an element in $\oplus_{k} \Omega_{*}^{\text {Spin }}\left(B U_{2 k}, \gamma_{2 k}\right) \otimes \mathbf{Q}$, we may assume that $\xi$ decomposes as a direct sum $\xi=\xi_{1} \oplus \xi_{2}$, where $\xi_{i}$ are odd dimensional complex vector bundles; moreover $\mathbf{C P}(\xi)$ is a Spin manifold.

Define

$$
\mu: S^{1} \times \mathbf{C P}\left(\xi_{1} \oplus \xi_{2}\right) \rightarrow \mathbf{C P}\left(\xi_{1} \oplus \xi_{2}\right)
$$

by

$$
\mu(\lambda,[v, w])=[\lambda v, w],
$$

for $\lambda \in S^{1}$, and $v$ and $w$ vectors in $\xi_{1}$ and $\xi_{2}$ respectively. This assignment is a semifree circle action with fixed point set $\mathbf{C P}\left(\xi_{1}\right) \cup \mathbf{C P}\left(\xi_{2}\right)$. By looking at codimensions, we see that $\mu$ is of odd type. Therefore $I_{*} \subset J_{*}$.

Denote by $\tau: \Omega_{*} \cap \mathbf{Q} \rightarrow \mathbf{Q}$ and by $\hat{A}: \Omega_{*} \otimes \mathbf{Q} \rightarrow \mathbf{Q}$ the ring homomorphisms sending each class $[M] \in \Omega_{*} \otimes \mathbf{Q}$ to its signature or $\hat{A}$-genus, respectively.

Proposition 3.2. The ideal $I_{*}$ is contained in $\operatorname{ker} \tau \cap \operatorname{ker} \hat{A}$.

Proof. For any complex $k$-plane bundle $\xi$, we have $\tau(\mathbf{C P}(\xi))=\tau\left(\mathbf{C} P^{k-1}\right) \cdot \tau(B)$, where $B$ is the base space of $\xi$ (see $[\mathbf{C}-\mathbf{H}-\mathbf{S}])$. Hence, for $k$ even, $\tau(\mathbf{C P}(\xi))=0$.

Next, from [A-H] (or [K] for semifree actions) $\hat{A}(M)=0$ if a connected Spin manifold $M$ admits a nontrivial circle action.

So $I_{*}$ is contained in $\operatorname{ker} \tau \cap \operatorname{ker} \hat{A}$.

If we want to find indecomposables in $\Omega_{*}^{\text {Spin }} \otimes \mathbf{Q} \cong \Omega_{*} \otimes \mathbf{Q}$ of the form $\mathbf{C P}(\xi)$, for $\xi$ a $2 k$-plane bundle, we must have nonzero Milnor numbers $\left\langle s_{2(n+2 k-1)}(c)(\mathbf{C P}(\xi)), \sigma_{\mathbf{C} P(\xi)}\right\rangle$ (see [M-S]). Here $2 n$ is the dimension of the base space of $\xi$. 
Let $\eta \rightarrow \mathbf{C} P^{2 n-3}$ be the canonical line bundle. Then it is not hard to see that for $\xi_{n}: \eta \otimes \eta \oplus \varepsilon^{3} \rightarrow \mathbf{C} P^{2 n-3}$, the Milnor numbers of $\mathbf{C P}\left(\xi_{n}\right)$ are nonzero if $n \geqslant 3$. Since the dimension of $\mathbf{C P}\left(\xi_{n}\right)$ is $4 n$, we succeed in finding indecomposables $x_{4 n}=\left[\mathbf{C P}\left(\xi_{n}\right)\right]$ of $\Omega_{*}^{\text {Spin }} \cap \mathbf{Q}$ for $n \geqslant 3$ which belong to $I_{*}$.

Also, if we let $x_{4}=\left[\mathbf{C} P^{2}\right]$ and $x_{8}=\left[\mathbf{H} P^{2}\right]$, we may write

$$
\Omega_{*}^{\text {Spin }} \otimes \mathbf{Q} \cong \Omega_{*}^{S O} \otimes \mathbf{Q}=\mathbf{Q}\left[x_{4}, x_{8}, \ldots, x_{4 n}, \ldots\right] \text {. }
$$

Hence, the ideal $\operatorname{ker} \tau \cap \operatorname{ker} \hat{A}$ in $\Omega_{*}^{S O} \otimes \mathbf{Q}$ is generated by $x_{4 n}, n \geqslant 3$, and the 16-dimensional element $x_{8}\left(x_{8}-x_{4}^{2}\right)$.

So, in the process of understanding the ideal $I_{*}$, we have obtained inclusions $\left(x_{12}, x_{16}, \ldots, x_{4 n}, \ldots\right) \subset I_{*} \subset\left(x_{8}\left(x_{8}-x_{4}^{2}\right), x_{12}, x_{16}, \ldots, x_{4 n}, \ldots\right)$.

We conjectured that as ideals of $\Omega_{*}^{\text {Spin }} \otimes \mathbf{Q}, I_{*}=\left(x_{12}, x_{16}, \ldots, x_{4 n}, \ldots\right)$.

Landweber and Stong (see [L-S]) obtained further characteristic numbers which vanish on the ideal $I_{*}$, the first one being $\left\langle\hat{\mathscr{A}}(M) \cdot \operatorname{ch}(T), \sigma_{M}\right\rangle$. We observe that this fact proves our conjecture up to dimension 20 .

As a matter of fact, the conjecture has been proved by S. Ochanine (see [O]). In his work he finds all multiplicative genera which vanish on the ideal $I_{*}$.

It is natural to ask if all characteristic numbers which vanish on $I_{*}$, continue to vanish if the action is not semifree. This is shown for the signature in $[\mathbf{L - S}]$; the general question appears to be quite difficult.

4. Free circle actions on spin manifolds. It remains to prove Propositions 2.2 and 2.3, and for this we must analyse in detail free $S^{1}$-actions of even and odd type on a connected Spin manifold. Let $Q$ denote the bundle of oriented frames over $M$ and let $(P, u)$ be the given Spin structure on $M$. Let $p: P \rightarrow M$ be the projection.

Proof of Proposition 2.2. Suppose first $\alpha$ is of even type. Then we have the following diagram:

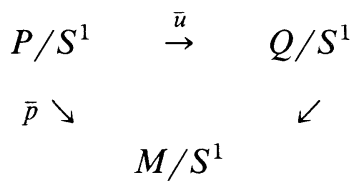

where $\bar{p}$ is a $\operatorname{Spin}(n)$-bundle and $\tau\left(M / S^{1}\right)=P / S^{1} \times_{\operatorname{Spin}(n)} \mathbf{R}^{n}$ (see [Sz]). Also, since $(\tau M) / S^{1}=\tau\left(M / S^{1}\right) \oplus \varepsilon$, the pair $(\bar{p}, \bar{u})$ defines a Spin structure on $M / S^{1}$.

To see that this Spin structure induces via $\pi: M \rightarrow M / S^{1}$ the given Spin structure on $M$, we consider the pull-back diagram:

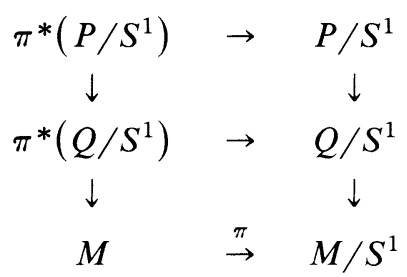

This, together with the maps $P \rightarrow P / S^{1}$ and $P \stackrel{p}{\rightarrow} M$, defines a map $\psi: P \rightarrow$ $\pi^{*}\left(P / S^{1}\right)$ which, under the identification $Q \cong \pi^{*}\left(Q / S^{1}\right)$, is an isomorphism. 
For the converse, we let $\bar{P}$ be a Spin structure on $M / S^{1}$ inducing via $\pi$ : $M \rightarrow M / S^{1}$ the Spin structure on $M$. Define $\bar{\alpha}: S^{1} \times \pi^{*}(\bar{P}) \rightarrow \pi^{*}(\bar{P})$ by $\bar{\alpha}(\lambda,(m, x))=(\alpha(\lambda, m), x)$, for $\lambda \in S^{1}$ and $(m, x) \in \pi^{*}(\bar{P})$. Then $\bar{\alpha}$ is a free circle action on $\pi^{*}(\bar{P})$ which commutes with the right $\operatorname{Spin}(n)$-action on $\pi^{*}(\bar{P})$ and is compatible with $\pi^{*}(\bar{P}) \rightarrow Q$. Therefore $\alpha$ is of even type.

Proof of Proposition 2.3. Assume first $\alpha$ is of odd type. Then the connected double covering $\hat{S}^{1}$ of $S^{1}$ acts freely on $P$.

\section{Define}

$$
\hat{\alpha}: P \times\left(\operatorname{Spin}(n) \times \hat{S}^{1}\right) \rightarrow P
$$

by

$$
\hat{\alpha}(x,(g, \lambda))=\lambda^{-1} x g,
$$

when $x \in P, g \in \operatorname{Spin}(n)$ and $\lambda \in \hat{S}^{1}$.

This is a free action inducing a free $\operatorname{Spin}^{c}(n)=\operatorname{Spin}(n) \times_{\mathbf{z}_{2}} \hat{S}^{1}$ action on $P$.

Also, the composition $P \stackrel{p}{\rightarrow} M \rightarrow M / S^{1}$ is a $\operatorname{Spin}^{c}(n)$-bundle over $M / S^{1}$. Define $\psi: P \rightarrow Q / S^{1} \times M$ by $\psi(x)=(\bar{\pi}(u(x)), p(x))$, where $\bar{\pi}: Q \rightarrow Q / S^{1}$; then, $\psi$ is equivariant and the diagram

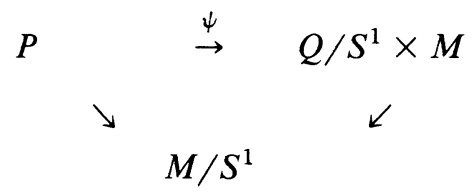

commutes. Therefore, we have a Spin ${ }^{c}$ structure on $Q / S^{1}$ and so on $\tau\left(M / S^{1}\right)$.

For the converse, we assume that the Spin structure on $M$ induces a $\operatorname{Spin}^{c}$ structure on $\tau\left(M / S^{1}\right)$ as in the diagram

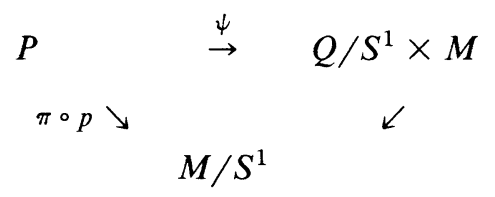

where $\psi(x)=(\bar{\pi}(u(x)), p(x)), x \in P$.

Consider the pull-back diagram:

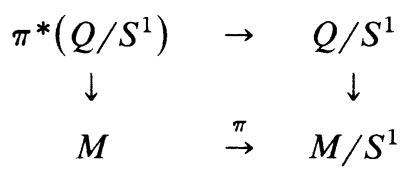

This, together with the maps $P \stackrel{\bar{\pi} \circ u}{\rightarrow} Q / S^{1}$ and $P \stackrel{p}{\rightarrow} M$, defines a map $v: P \rightarrow$ $\pi^{*}\left(Q / S^{1}\right)$ given by $v(x)=(\bar{\pi}(u(x)), p(x))$. Since $\psi$ is a double covering, the diagram

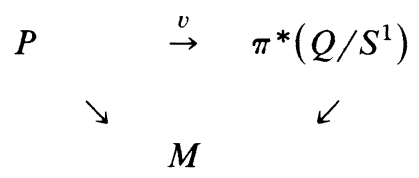


defines a Spin structure on $M$, which, under the identification $\pi^{*}\left(Q / S^{1}\right) \cong Q$, coincides with the given one.

To see that $\alpha$ is of odd type, we let $x \in P$ and the family of maps $\alpha_{t}$ : $\pi^{*}\left(Q / S^{1}\right) \rightarrow \pi^{*}\left(Q / S^{1}\right)$ given by $\alpha_{t}(v(x))=\left(\bar{\pi}(u(x)), \alpha\left(e^{2 \pi i t}, p(x)\right)\right), t \in \mathbf{R}$, induced by $\alpha$. Define

$$
\tilde{\alpha}_{t}: \pi^{*}\left(Q / S^{1}\right) \rightarrow P
$$

by

$$
\tilde{\alpha}_{t}(v(x))=x \cdot\left[1, e^{\pi i t}\right],
$$

where $\left[1, e^{\pi i t}\right] \in \operatorname{Spin}^{c}(n)$. Then

$$
v\left(\tilde{\alpha}_{t}(v(x))\right)=v \cdot\left[x, e^{\pi i t}\right]=\psi\left(x \cdot\left[1, e^{\pi i t}\right]\right)=\alpha_{t}(v(x)),
$$

$\tilde{\alpha}_{0}(v(x))=x$ and $\tilde{\alpha}_{1}(v(x))=x \cdot[1,-1]$. Hence $\alpha$ is of odd type.

\section{REFERENCES}

[A-B] M. F. Atiyah and R. Bott, A Lefschetz fixed point formula for elliptic complexes, II. Applications, Ann. of Math. (2) 88 (1968), 451-491.

[A-H] M. Atiyah and F. Hirzebruch, Spin-manifolds and group actions, Essays on Topology and Related Topics, Springer, 1970, pp. 18-28.

[B] L. D. Borsari, Bordism groups of semi-free circle actions on Spin manifolds, Ph.D. thesis, Rutgers Univ., October 1985.

[C] P. E. Conner, Differentiable periodic maps, Lecture Notes in Math., vol. 738, Springer-Verlag, 1979.

[C-F] P. E. Conner and E. E. Floyd, Torsion in SU-bordism, Mem. Amer. Math. Soc. No. 60, 1966.

[C-H-S] S. S. Chern, F. Hirzebruch and J. P. Serre, On the index of a fibered manifold, Proc. Amer. Math. Soc. 8 (1957), 587-596.

[K] Y. Kitada, Semi-free circle actions on Spinc-manifolds, RIMS(Kyoto) 10 (1974), 601-607.

[L-S] P. Landweber and R. E. Stong, Circle actions on Spin manifolds and characteristic numbers (to appear).

[L-W] C. Lazarov and A. G. Wasserman, Free actions and complex cobordism, Proc. Amer. Math. Soc. 47 (1975), 215-217.

[M-S] J. W. Milnor and J. D. Stasheff, Characteristic classes, Princeton Univ. Press, Princeton, N. J., 1974.

[O] S. Ochanine, Sur les genres multiplicatifs défini par des intégrales (to appear).

[St] R. E. Stong, Notes on cobordism theory, Math. Notes, Princeton Univ. Press, Princeton, N. J., 1968.

[Sz] R. H. Szczarba, The tangent bundle of fibre spaces and quotient spaces, Amer. J. Math. 86 (1964), 685-697.

[U] F. Uchida, Cobordism groups of semi-free $S^{1}$ - and $S^{2}$-actions, Osaka J. Math. 7 (1970), 345-351.

[W] E. Witten, Fermion quantum numbers in Kaluza-Klein theory, Shelter Island II: Proc. 1983 Shelter Island Conf. on Quantum Field Theory and the Fundamental Problems of Physics (Eds., R. Jackin, N. N. Khari, S. Weinberg and E. Witten), MIT Press, Cambridge, Mass., 1985, pp. 227-277.

Department of Mathematics, Rutgers University, New Brunswick, New Jersey 08903

Current address: Instituto de Matemática e Estatistica, Universidade de São Paulo, Cidade Universitária “Armando Salles de Oliveira”, Caixa Postal no. 20570 (Agencia Iguatemi), CEP 01498, São Paulo, Brasil 